\title{
MICROMACHINED DUAL INPUT AXIS ANGULAR RATE SENSOR
}

\author{
Thor Juneau, A. P. Pisano \\ Berkeley Sensor \& Actuator Center, 497 Cory Hall \\ University of Califomia at Berkeley \\ Berkeley, CA 94720 \\ e-mail: thorj@nobelium.me.berkeley.edu
}

\begin{abstract}
The need for inexpensive yet reliable angular rate sensors in fields ranging from automotive to consumer electronics has sparked prolific micromachined angular rate sensor research. The vast majority of research has focused on single input axis angular rate sensors based upon either translational resonance, such as tuning forks, or structural mode resonance, such as vibrating rings. However, this work presents an entirely novel approach based on angular resonance of a rotating rigid rotor suspended by torsional springs. The inherent symmetry of the circular design allows angular rate measurement about two axes simultaneously. Using a commercial micro-accelerometer production process, two versions of a fully integrated, $660 \mu \mathrm{m}$ diameter dual input axis angular rate sensor have been designed, fabricated, and tested. Minimum detectable signals as small as $1.2 \% \mathrm{sec}$ over a $25 \mathrm{~Hz}$ bandwidth were achieved with untuned rate sensors having mismatched mode frequencies. However, with mode frequency tuning the performance was improved to $10 \% \mathrm{~min}$ over an equivalent bandwidth.
\end{abstract}

\section{INTRODUCTION}

The advent of micromachining has raised the possibility of fabricating high performance sensors without the associated high costs. In particular, there has been great interest in manufacturing micromachined angular rate senors [1]. Until now, the high cost of even medium performance rate sensors has precluded their use in most consumer products and high volume military applications, such as automatic vehicle braking systems, automobile navigation, camera stabilization, and artillery munitions guidance.

A number of angular rate sensors have been recently reported, but the majority of these designs are single axis sensors based on translational vibration [2] or structural mode vibration [3]. In addition, many reported rate sensors require fabrication processing not compatible with standard VLSI processes. In contrast, this research has focused on surface micromachining a dual input axis angular rate sensor in the fully integrated Analog Devices Inc. (ADI) microaccelerometer production process [4]. This proven fabrication process allowed inexpensive production with integrated electronics to improve noise performance. Unlike most previous designs, the dual axis rate sensor presented here is based on rotational resonance of a polysilicon disk. Since the disk is symmetric in two orthogonal axes, the sensor can sense rotation equally well about both these two orthogonal axes. When combined with a $\mathrm{z}$-axis rate sensor [5] or [3], the angular rate about all three axes can be measured using one die.

\section{BASIC OPERATING PRINCIPLE}

The micromachined dual input axis angular rate sensor is composed of an inertial rotor, an actuation means to drive this inertial rotor into resonance, and a sensing system to measure angular deflections of the inertial rotor due to Coriolis accelerations. The inertial rotor is a $2 \mu \mathrm{m}$ thick polysilicon disk with a $150 \mu \mathrm{m}$ radius. As depicted in Fig. 1, this inertia rotor is suspended on four symmetrically placed beams anchored to the substrate. These beams provide a torsional suspension for the rotor allowing compliance about three mutually perpendicular axes. To achieve maximum mechanical sensitivity, the suspension was designed to match the frequencies of all three rotational modes sufficiently well so that electrostatic tuning may compensate for fabrication process variation.

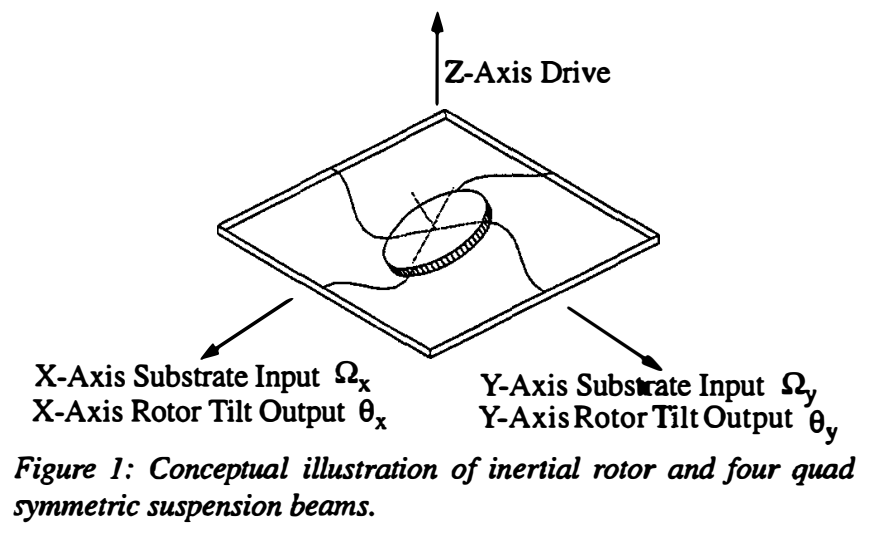

The inertial rotor is driven at angular resonance about the zaxis (perpendicular to the substrate) using the electrostatic comb drive described in a later section. The resonating inertial rotor is responsive to substrate rotation rates about both the $x$ - and $y$-axes parallel to the substrate as defined in Fig. 1. A rotation rate about the $x$-axis induces a Coriolis angular oscillation about the $y$-axis, and likewise a rotation rate about the $y$-axis induces a Coriolis angular oscillation about the $\mathrm{x}$-axis. Sensing these Coriolis oscillations and relating them to the original substrate rate inputs forms the foundation of angular rate sensor operation.

The Coriolis angular oscillation or tilting about each sense axis is embodied in the dynamical equation of motion below where $\theta_{x}$ is the sense axis tilt angle, $I_{x x}$ is the moment of inertia, $C_{x}$ is the damping coefficient, and $K_{x}$ is the spring constant all reckoned about the $x$-axis. Only the $x$-axis is given since the equation for the $y$-axis is essentially identical except $x$ and $y$ subscripts are reversed and the right hand side is positive.

$$
I_{x x} \ddot{\theta}_{x}+C_{x} \dot{\theta}_{x}+K_{x} \theta_{x}=-I_{z z} \Omega_{y} \times \theta_{z 0} \omega_{z} \operatorname{Cos}\left(\omega_{z} t\right)
$$

The term on the right hand side is the Coriolis acceleration due to conservation of angular momentum. The angular rate input about the y-axis to be measured is $\Omega_{y}$. This is multiplied by the $z$ axis moment of inertia $\mathrm{I}_{\mathrm{zz}}$ and the resonating $\mathrm{z}$-axis angular rate which is an $\omega_{z}$ frequency cosine with amplitude $\theta_{z 0} \omega_{z}$. Hence the Coriolis acceleration and the ensuing Coriolis angular oscillation of the rotor both can be thought of as amplitude modulated signals with a carrier frequency $\omega_{z}$. The amplitude of this modulated Coriolis oscillation is directly proportional to the y-axis input rate $\Omega_{y}$ This Coriolis oscillation is sensed using the change in capacitance between the rotor and four sense electrodes beneath the inertial rotor. Since the Coriolis oscillation is in phase with the 
resonating z-axis angular rate, simply demodulating the sense signal with the resonant drive signal yields the desired rate measurement. The dynamics of the second sense axis are similar.

The fact that the Coriolis oscillation is in phase with the drive forcing signal is of paramount importance. Unfortunately due to fabrication imperfections, the disk will wobble slightly in phase with resonance position. The wobble can masquerade as a false rate measurement called quadrature error [5] and can be larger than $200 \% \mathrm{sec}$. Ideally, the final demodulation with the drive signal will eliminate the quadrature error from the measurement because the quadrature error is $90^{\circ}$ out of phase. However, in practice small phase lags $\left(-0.5^{\circ}\right)$ in the electronics allow a small percentage of quadrature enror to corrupt the measurement. Such factors as the performance required, the lag of the electronics, and the stability of resonant amplitude dictate whether the DC offset due to quadrature error may be tolerated or require active cancellation. With the basic operation described, attention is now focused on specifics.

\section{MECHANICAL STRUCTURE}

The heart of the dual input axis angular rate sensor is the micromachined structure itself. It is fabricated in true surface micromachining tradition by using a structural polysilicon layer deposited over a sacrificial oxide and patterned [4]. Etch holes in the sacrificial oxide provide anchor points to the underlying substrate after the sacrificial oxide is removed. The combined inertial rotor and spring suspension were carefully designed to satisfy many design constraints placed on the natural frequencies of the structure. For example, natural frequencies in the upper audio range are desirable for shock and random vibration robustness. The rate sensor should be insensitive to all translational accelerations, so the natural frequency of all three translation directions must be high. In this design, the $\mathrm{x}$-axis and $\mathrm{y}$-axis directions parallel to the substrate are very stiff. Although the $\mathrm{z}$-axis perpendicular to the substrate is not nearly as stiff (approx. $15 \mathrm{kHz}$ ), the differential sensing scheme discussed later nulls z-axis translation sensitivity to first order. Ideally, the drive natural frequency and the pair of sense natural frequencies should be designed to be nearly equal. However, process variation will result in mismatch unless electrical tuning is used to artificially match frequencies. Electrostatic forces due to voltage differentials are always attractive, hence spring constants and natural frequencies can only be decreased. For this reason, the sense axis natural frequencies were designed to be $10 \%$ above the drive natural frequency so that they may be down tuned to match the drive frequency. This is implemented by using spring suspension beams with a cross-section of $2 \mu \mathrm{m}$ thick by $1.8 \mu \mathrm{m}$ wide with the thicker dimension stiffening the sense axis spring constants.

With the above constraints in mind, two distinct rate sensor versions were fabricated. Each version has identical rotor sizes, but different suspension designs. A compliant serpentine suspension shown in Fig. 2 gave a low natural frequency of $21 \mathrm{kHz}$. The main spring suspension beam is $180 \mu \mathrm{m}$ in length, while the shorter serpentine beams are $120 \mu \mathrm{m}$ in length. The second version has a less compliant suspension composed of a single $180 \mu \mathrm{m}$ beam with some stress relief (as shown later in Fig. 6) which gave a higher natural frequency of $28.6 \mathrm{kHz}$. In each version, an external suspension extends from the outside of the inertial rotor. This suspension design can accommodate warping of the inertial rotor due to large residual stress gradients in the polysilicon [6].

\section{ELECTROSTATIC RESONANT DRIVE}

There can be no rate sensing unless the inertia rotor is in mocion. To this end, the inertial rotor is driven into rotational resonance about the $z$-axis perpendicular to the substrate. Electrostatic comb drive was used as it has the distinct advantage of remaining linear despite large displacements. However, this technique requires that the angular rate sensor be operated with ambient pressure below $150 \mathrm{mTorr}$.

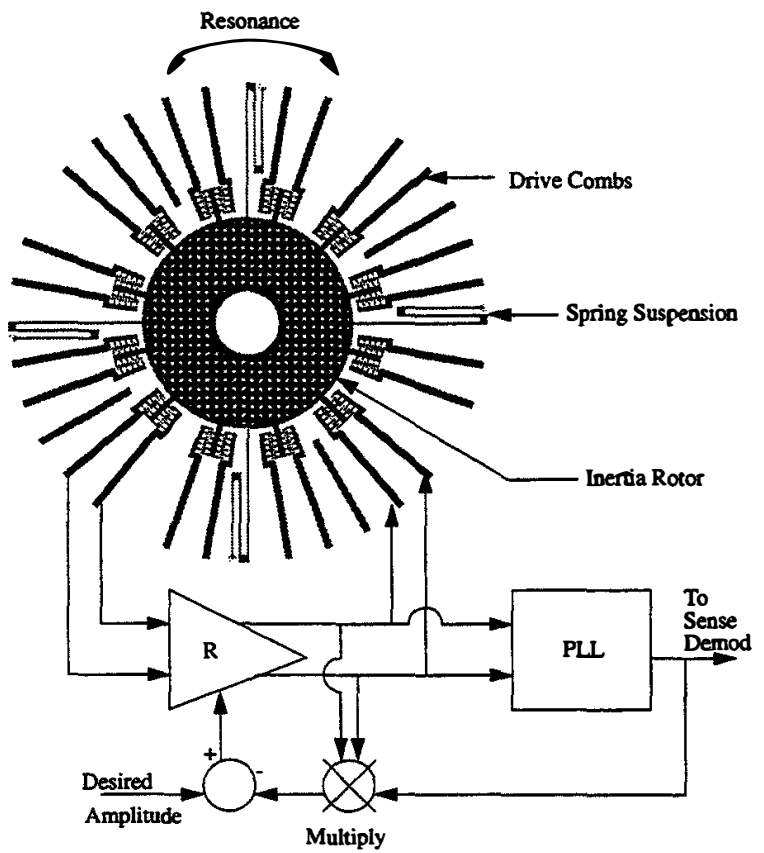

Figure 2: Diagram of polysilicon dual input axis rate sensor looking down on die. Schematic shows trans-resistance resonant drive with automatic gain control to hold oscillation amplitude constant plus phase-lock-loop for Coriolis demodulation.

As discussed in Nguyen [7] a trans-resistance amplifier provides positive feedback which induces resonance. The amplifier and combs (see Fig. 2) were designed to ensure adequate forcing to overcome air damping. This design used a differential drive comprised of 60 comb teeth and requiring approximately $3 \mathrm{M} \Omega$ drive with a 10 volt difference between the inertial rotor and drive combs. In addition, the driving circuitry has low phase lag and feedthrough to reduce the effect of quadrature error. The resonant amplitude is carefully controlled using automatic gain control because scale factor is directly proportional to resonant amplitude. A phase-lock-loop provides a clean signal for Coriolis signal demodulation discussed next.

\section{DUAL AXIS CORIOLIS OSCILLATION SENSING}

The inerial rotor tilts due to Coriolis acceleration and this Coriolis oscillation is measured by sensing the change in capacitance between the inertial rotor and the four $n+$ diffusion sense electrodes beneath the inertial rotor. As shown in Fig. 3, when a differential modulation voltage is applied to a pair of diametrically opposed sense electrodes (each shaped as a quarter-pie section), a rotation of the rotor about the sense axis of these electrodes produces a voltage on an integrator electrically connected to the rotor. Since the pair of sense electrodes provides differential 
sensing, any translation of the rotor up or down along the z-axis direction produces no change in sense output. Dual axis operation is achieved by using a different voltage modulation frequency $(200 \mathrm{kHz}$ and $300 \mathrm{kHz})$ for each pair of sense electrodes. Separate demodulation circuits for each sense axis provide two voltage outputs proportional to the two angular rate inputs. The output voltages must be demodulated twice: first a demodulation removes the high sense modulation frequency, and second a demodulation removes the drive resonant frequency leaving the base band rate input measurements.

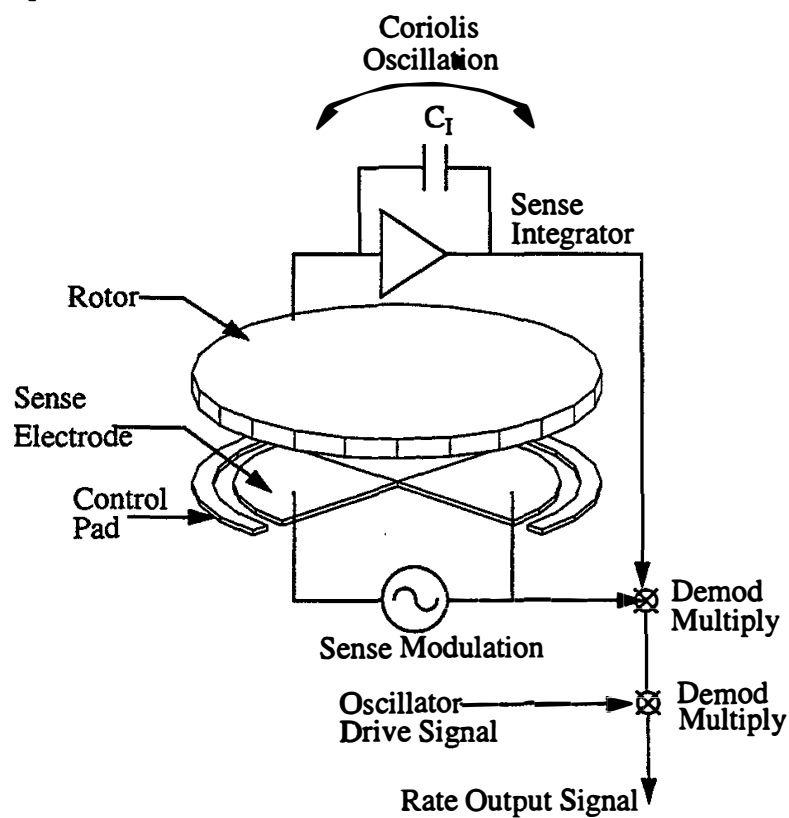

Figure 3: Conceptual side view showing rotor with underlying diffusion sense electrodes for one axis. Please note only two of four sense electrodes are shown for simplicity.

The afore mentioned single ended integrator scheme (using an integrating capacitor $\mathrm{C}_{\mathrm{I}}$ of $50 \mathrm{fF}$ ) was used for two principle reasons. First, the structure itself has far less noise producing parasitic capacitance than the $n+$ diffusion electrodes. Second, the integrator improves linearity [5]. Absence of distortion in the sense circuitry is critical, for any odd harmonic distortion can lead to quadrature error signals mixing with true Coriolis measurement signals. One drawback of sensing on the driven structure is that despite fully differential comb drive, a small inherent voltage at twice the drive frequency appears on the integrator. In general, this signal is small and is far from the crucial sense modulation frequencies.

\section{SENSITIVITY}

The mechanical sensitivity relating Coriolis rotor oscillation amplitude to substrate angular rate input for each sense axis can be derived from Eq. 1 with the final form shown below for the $\mathrm{x}$-axis.

$$
\left|\begin{array}{c}
\theta_{x} \\
\Omega_{y}
\end{array}\right|=\left|\frac{2 \theta_{z 0} \omega_{z}}{\omega_{x}^{2}+\frac{j \omega_{x} \omega_{z}}{Q_{x}}-\omega_{z}^{2}}\right|
$$

This is the standard second order frequency response with $\theta_{\mathrm{z} 0}$ the resonant drive amplitude, $\omega_{z}$ the resonant drive frequency, $\omega_{x}$ the sense axis frequency, and $Q_{x}$ the quality or inverse damping coefficient. Since the rate sensor is operated in partial vacuum, the quality factor $Q_{x}$ is generally above 800 . Thus, a large resonant peak exists which provides excellent gain when drive and sense frequencies match.

The combined mechanical and electrical sensitivity must also be considered since mechanical sensitivity alone can be misleading. The combined mechanical system, sense electrodes, and integrator can be summarized as below with sense voltage $V_{s}$, integrating capacitor $C_{\mathrm{I}}$, and electrode capacitive sensitivity $\partial C / \partial \theta_{x}$.

$$
\left|\frac{V_{\text {out }}}{\Omega_{y}}\right|=\left|\frac{\theta_{x}}{\Omega_{y}}\right|\left|\frac{V_{\text {out }}}{\theta_{x}}\right|=\left|\frac{2 \theta_{z 0} \omega_{z}}{\omega_{x}^{2}+\frac{j \omega_{x} \omega_{z}}{Q_{x}}-\omega_{z}^{2}}\right|\left|2 \frac{\partial C}{\partial \theta_{x}} \frac{V_{s}}{C}\right|
$$

Clearly increasing the sense voltage differential $\mathrm{V}_{\mathrm{s}}$ between the rotor and the quarter circle sense elecrodes will increase overall sensitivity. Unfortunately, this sense voltage also results in an electrostatic force which tends to pull the inertial rotor down to the substrate. The critical pull down voltage limits the maximum applicable sense voltage and is proportional to the natural frequency for $\mathrm{z}$-axis ranslation perpendicular to the substrate. For this reason, the sense voltage for weaker suspensions must be reduced, nearly eliminating all mechanical sensitivity gains. However, the sense voltage also has another side effect which complicates simple sensitivity generalizations. The sense voltage down tunes the sense axes natural frequencies as discussed in the following section.

\section{FREQUENCY TUNING}

The voltage applied between the inertial rotor and the four quarter circle sense electrodes results in an attractive electrostatic force which down tunes the natural frequencies of both the rotational sense axes. This frequency tuning can be used to nearly match frequencies and vastly improve sensitivity. The tuning range is limited because any tuning voltage must be below the critical pull down voltage. Hence, the design target values for the drive mode and the sense mode frequencies must be far enough apart to accommodate process variation, but not so far apart that they are outside the tuning range.

Under open-loop operation, matching natural frequencies exactly has several drawbacks. Because the gain and phase change radically at the resonant peak, gain is not constant over the bandwidth, frequency drift cause large scale factor changes, and the phase swings from 0 to 180 degrees. Cross-sensitivity is also compromised as large resonant peak gained signals many crosscouple. Therefore during open-loop operation it is advantageous to match modes only within $2-5 \%$. On the other hand, the use of closed-loop feedback should alleviate most the these difficulties.

\section{EXPERIMENTAL RESULTS WITHOUT TUNING}

Two versions of the dual input axis angular rate sensor were fabricated using the ADI surface micromachining processes. In addition to performance, those parameters with greatest impact on performance, such as natural frequency, quality factor $Q$, and maximum sense voltage, have been experimentally identified. The two versions have identical inertial rotor sizes but different suspensions giving drive natural frequencies of $28.6 \mathrm{kHz}$ and $21 \mathrm{kHz}$. Both sensors were tested in 60 mTorr vacuum which gave the high frequency version a quality factor $Q$ of 960 and the low frequency 
version a $\mathrm{Q}$ of 820 . The pull down voltage of the high frequency sensor was 4.2 volts, whereas the low frequency sensor could withstand only 3.4 volts.

The performance results for the rate sensors as fabricated without tuning are summarized in Fig. 4. Due to process variation, a distribution of nearly matched and unmatched rate sensors could be investigated. The dual input axis angular rate sensor having mode mismatch of $2 \%$ provided far better performance with $2 \mathrm{mV}$ per \% sec sensitivity and a $0.24 \% / \mathrm{sec} / \sqrt{\mathrm{Hz}}$ noise floor limited by the electrical noise of the integrator input transistor. Thus, a minimum detectable signal of approximately $1.2 \% \mathrm{sec}$ over a $25 \mathrm{~Hz}$ bandwidth was achieved.

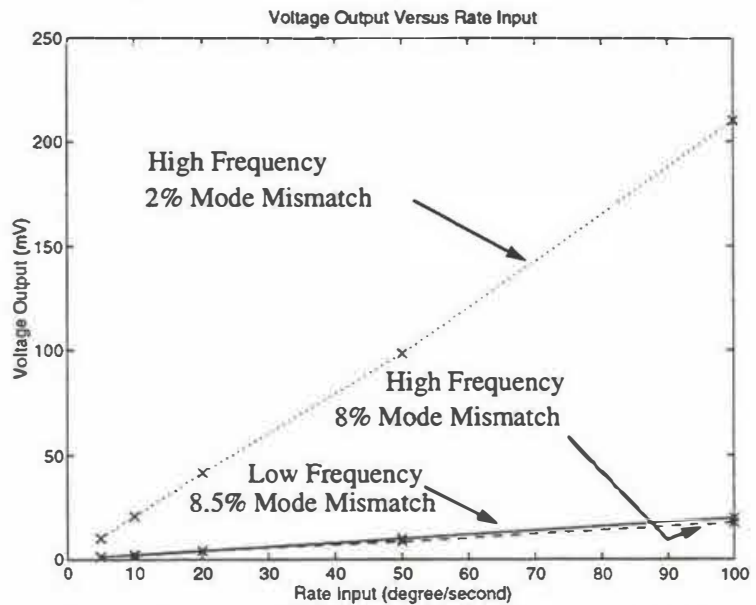

Figure 4: Graph showing rate sensor voltage amplitude output versus rate amplitude input for a $1 \mathrm{~Hz}$ sinewave.

\section{EXPERIMENTAL RESULTS WITH TUNING}

Due to the high quality factor $\mathrm{Q}$, great performance improvement may be reaped using electrostatic tuning. As displayed in the top graph of Fig. 5, increasing voltage between the quarter circle sense electrodes and the rotor down tunes the sense frequency more than $2500 \mathrm{~Hz}$, while down tuning the drive frequency only slightly.
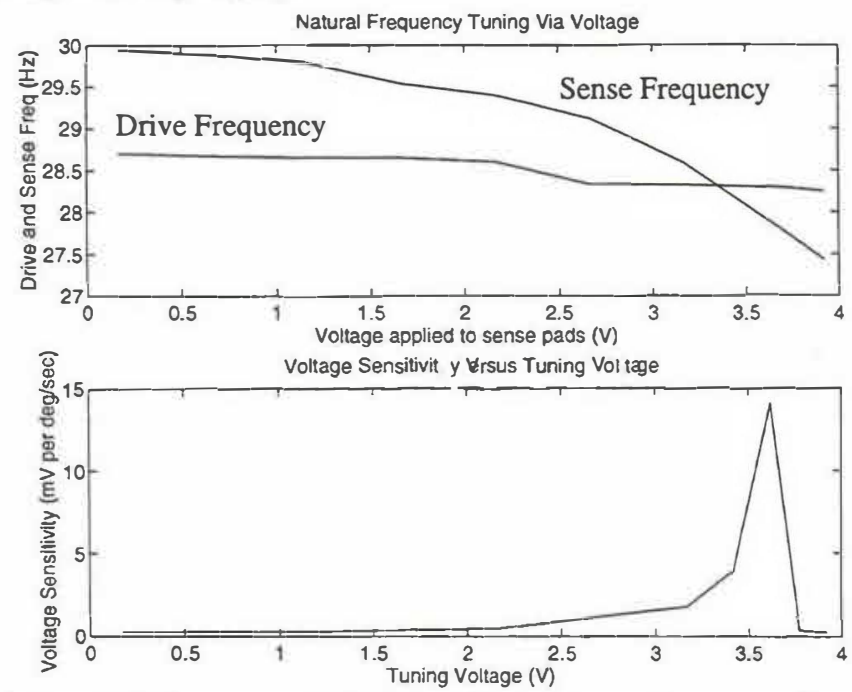

Figure 5: The top graph shows drive and sense frequency down tuning due to applied voltage. The lower graph shows the dramatic impact frequency matching has on sensitivity.
The bottom graph in Fig. 5 shows sensitivity is maximized roughly at the point where the frequencies match with some offset due to experimental error. Tuning resulted in performance of $10 \%$ min over a $25 \mathrm{~Hz}$ bandwidth.

\section{CONCLUSION}

The underlying operating principles and resulting design methodology for a dual input axis angular rate sensor based on angular resonance were presented. Fabricated rate sensors (as shown in Fig. 6) without tuning provided good noise performance. However, electrostatic frequency tuning improved performance significantly.

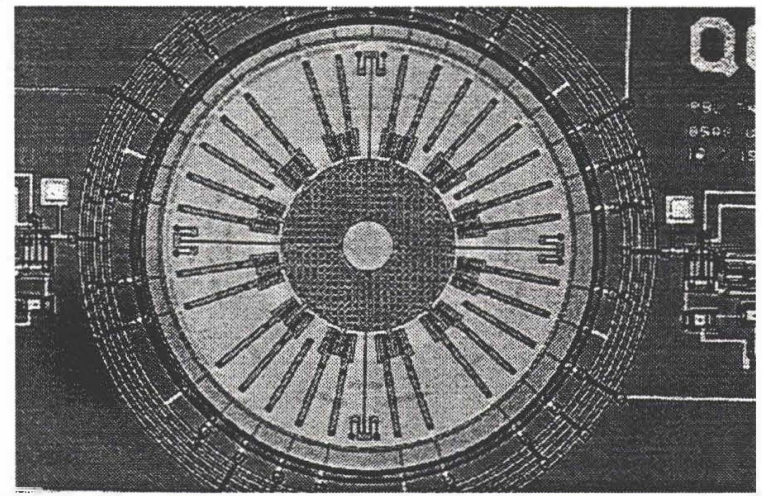

Figure 6: Close-up die photo showing high frequency version. Note that the beam suspension is not serpentine.

\section{ACKNOWLEDGEMENTS}

First, the authors must thank ADI for all device fabrication using the excellent ADI surface micromachining process. Also, without the financial support of both ARPA (grant TP-26890) and the Califomia PATH project, this research could not continue. The insights and guidance of Professor $R$. T. Howe have been invaluable. The first author also must thank Per Ljung for his previous rate sensor work. However, the most heart felt thanks on behalf of the first author goes to Bill Clark for his help with key circuitry.

\section{REFERENCES}

1. J. Soderkvist, "Micromachined Gyroscopes", Sensors and Actuators A, pp. 65-71 (1994 v43).

2. J. Bernstein and M. Weinberg, "A Micromachined Comb-drive Tuning Fork Rate Gyroscope", 21st Joint Serv. Data Exchng for G. N. and C., Palm Springs, CA, Oct. 1992.

3. M. Putty and K. Najafi, "A micromachined Vibrating Ring Gyroscope", 1994 Solid-State Sensor and Actuator Workshop, Hilton Head, pp. 213.

4. R. S. Payne, S. Sherman, S. Lewis, R. T. Howe, "Surface Micromachining: From Vision to Reality to Vision", 1995 IEEE International Solid State Circuits Conference, pp. 164.

5. W. A. Clark, R. T. Howe, R. Horowitz, "Surface Micromachined Z-Axis Vibratory Rate Gyroscope", 1996 Solid-State Sensor and Actuator Workshop, Hilton Head.

6. P. B. Ljung, T. Juneau, A. P. Pisano, "Micromachined two input axis angular rate sensor", ASME International Mechanical Engineering Congress and Exposition, session DSC-16, (1995).

7. C. H. Nguyen, "Micromachanical Signal Processors", Ph.D. Thesis, University of California at Berkeley, Berkeley, CA 1994. 